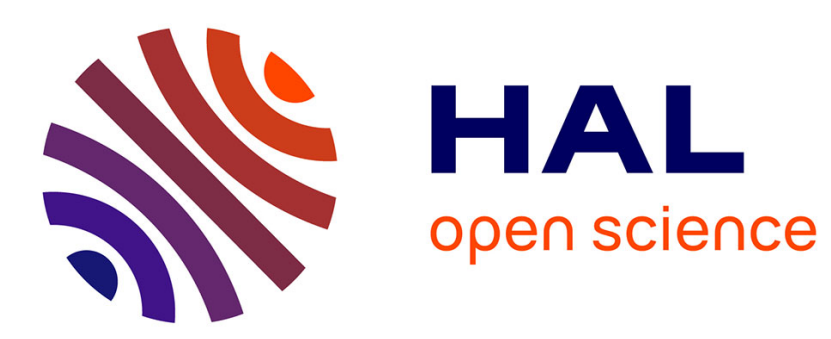

\title{
Paint-on-Glass Animation: The Fellowship of Digital Paint and Artisanal Control
}

Tom van Laerhoven, Fabian Di Fiore, William van Haevre, Frank van Reeth

\section{To cite this version:}

Tom van Laerhoven, Fabian Di Fiore, William van Haevre, Frank van Reeth. Paint-on-Glass Animation: The Fellowship of Digital Paint and Artisanal Control. Computer Animation and Virtual Worlds, 2011, 22 (2-3), pp.325. 10.1002/cav.406 . hal-00629936

\section{HAL Id: hal-00629936 https://hal.science/hal-00629936}

Submitted on 7 Oct 2011

HAL is a multi-disciplinary open access archive for the deposit and dissemination of scientific research documents, whether they are published or not. The documents may come from teaching and research institutions in France or abroad, or from public or private research centers.
L'archive ouverte pluridisciplinaire HAL, est destinée au dépôt et à la diffusion de documents scientifiques de niveau recherche, publiés ou non, émanant des établissements d'enseignement et de recherche français ou étrangers, des laboratoires publics ou privés. 


\section{Paint-on-Glass Animation: The Fellowship of Digital Paint and Artisanal Control}

\begin{tabular}{|r|l|}
\hline Journal: & Computer Animation and Virtual Worlds \\
\hline Manuscript ID: & CAVW-11-0018 \\
\hline Wiley - Manuscript type: & Special Issue Paper \\
\hline Author: & 04-Mar-2011 \\
\hline Complete List of Authors: & $\begin{array}{l}\text { Van Laerhoven, Tom; Hasselt University, Expertise Centre for } \\
\text { Digital Media } \\
\text { Di Fiore, Fabian; Hasselt University, Expertise Centre for Digital } \\
\text { Media } \\
\text { Van Haevre, William; Hasselt University, Expertise Centre for Digital } \\
\text { Media } \\
\text { Van Reeth, Frank; Hasselt University, Expertise Centre for Digital } \\
\text { Media }\end{array}$ \\
\hline Keywords: & $\begin{array}{l}\text { animation, 2D animation, painterly animation, paint system, paint- } \\
\text { on-glass }\end{array}$ \\
\hline $\begin{array}{l}\text { Note: The following files were submitted by the author for peer review, but cannot be converted } \\
\text { to PDF. You must view these files (e.g. movies) online. }\end{array}$ \\
\hline CASA2011_\#77_figures.zip \\
\hline
\end{tabular}




\title{
Paint-on-Glass Animation
}

\section{The Fellowship of Digital Paint and Artisanal}

\author{
Control \\ Tom Van Laerhoven Fabian Di Fiore William Van Haevre \\ Frank Van Reeth \\ Hasselt University - tUL - IBBT \\ Expertise Centre for Digital Media \\ Wetenschapspark, 2 \\ BE-3590 Diepenbeek, Belgium \\ \{tom.vanlaerhoven, fabian.difiore, william.vanhaevre, frank.vanreeth\}@uhasselt.be \\ http: / / www . edm. uhasselt.be
}

\author{
Abstract \\ In this paper we deal with paint-on-glass animation, which is a technique for mak-


ing animated films by pushing slow-drying paints from frame to frame directly under the camera. As artwork is continuously destroyed upon creating new frames, the animator is not able to rehearse or refine the animation afterwards. Furthermore, due to impracticable issues like how to stack up layers containing wet paint or how to overlay masks on the wet medium, one has to take both creative as technical decisions for each shot.

Our approach consists of an interactive paint setup that physically simulates paint media. Together with a set of digital tools the artist is relieved from the difficult task of sustaining a constant frame-to-frame coherence while animating and is given the possibility to modify or undo earlier paint modifications. Regarding the interaction part, the artist stays in full control by employing a tangible interface. This allows artists to push pigment with their fingers, use special-purpose digital brushes, or even to employ real life tools like paper tissue.

Feedback from artists confirms the real-life behaviour of stretching and smudging the paint as well as interacting with the setup, resulting in a natural and reality-based methodology.

Keywords: animation, 2D animation, painterly animation, paint system, paint-on-glass 


\section{Introduction}

In spite of its name, paint-on-glass animation is best defined as a technique for making animated films by pushing around some kind of (wet) medium directly under the camera and recording it frame-by-frame (Figure 1). It is categorised as an "under-the-camera-technique" as it is nearly always undertaken by an individual artist or animator rather than by a production studio using factory-like processes. As the medium is pushed around directly under the camera and recorded frame-by-frame, each image seems to merge from the previous one and melt into the next, resulting in movement that can be very fluid and organic - a continual process of metamorphosis.

A well-known practitioner of the paint-on-glass animation technique is Russian animator Aleksander Petrov. His most famous work is the award winning short film "The Old Man and the Sea" which took two and a half years to complete [1] illustrating the complexity of the technique.

Motivation. As the medium is pushed around from frame to frame, artwork is continuously destroyed as new artwork is created. Without the ability to rehearse and refine the animation and without the disposal of layering and masking tools, the animator must plough on regardless incorporating any errors into the sequence.

On the one hand this stimulates spontaneity and delivers work that is very fresh and distinctive as it celebrates the method of its making. For example, characters may move from place to place not by walking, but by being smudged away to re-form out of the background 
at the required location. On the other hand, this particular form of step-ahead animation is a painstaking process which demands much self-discipline, endurance and concentration as the resulting frames are never a series of clean images of the kind produced by other animation techniques, but also contain a record of their making due to the pushing process.

Contribution. In this paper we present our approach to facilitate paint-on-glass animation. In close collaboration with artists and animators we developed a set of digital tools that do enable them to achieve the fine quality of a finished painting for each frame, while preserving all artisan control.

Primarily, our system features:

- a real-time free-form paint tool to physically simulate the complex behaviour of different paint media (gouache, watercolour, impasto, pastel);

- painting/animating by pushing the drawing media around resulting in a stretch and smear effect;

- a tangible interface consisting of a multitouch screen allowing the use of one's fingers as well as physical media including brushes and tissues;

- several tools that are absent in the traditional method due to the wet nature of the painting media such as working with layers and masks;

- the availability of animation tools like onion skinning and flipbook animation; 
- digital tools to control the drying time, correct mistakes, remove pigment and/or water as desired, and the ability to save, load or reuse (intermediate) results.

Paper Organisation. First, we survey work we consider related to ours. Next, we describe our approach starting from the employed painting setup to an overview of the digital painton-glass animation process. Finally, clarifying examples are provided in which we also discuss our results.

\section{Related Work}

We start this section by giving a brief overview of traditional paint-on-glass animation. Then, we elaborate on digital paint solutions that we consider related to ours.

\section{Traditional Paint-on-Glass Animation}

What we give here is an account as it might be for a practiced artist in a home studio. We separately discuss the painting process (i.e. painting a single frame) and the animation process (i.e. making a series of drawings).

Painting. The painting process itself resembles painting on a canvas as the initial paint is added with brushes or other tools including sponges, cotton buds, small sticks, strong tissue or even fingers. 
As the paint medium will be reused from frame to frame, the artist is constrained to work with slow drying paint media like oil paint in order to keep working with the medium across several hours or even days. This causes also many impracticable issues like how to stack up layers of wet paint or how to overlay masks on the wet medium.

Animating. For classic 2D animation the animator draws or sets up objects one frame at a time in sequential order until the sequence is complete, also referred to as step-ahead animation. In this way there is one physical drawing or image per frame. In the case of paint-on-glass animation the artist is working directly under-the-camera: the artist has to record the current frame before proceeding to the next one as current artwork is continuously destroyed for creating each new frame. When a sequence has been filmed, there is no raw material left besides the final film. So, while animating one cannot rely on techniques like onion skinning or flipbook animation as previous artwork is not available anymore. Hence, there is also no artwork to turn to when modifying the film afterwards.

\section{Digital Paint Systems}

In this section we will discuss digital paint solutions that we consider related to ours.

Painting. Looking at the majority of published paint systems [2, 3, 4, 5], virtual brushes are by default operated by some kind of digital stylus in combination with a digital tablet. Although popular and basically intuitive, this way of working is nothing more than an ap- 
proximation of 'the real thing'. Issues such as parallax, the lack of tufts and absence of physical tools need to be solved first.

Other paint systems explicitly target novel interfaces to bridge the gap between physical and digital painting. Vandoren et al. developed IntuPaint, which is a tangible interface for a digital paint easel, using an interactive surface and electronic brushes with a tuft of bristles [6]. This approach provides natural interaction and enables detailed tracking of specific brush strokes. Additional tangible and finger-based input techniques allow for specific paint operations or effects. Okaichi et al. propose an interactive oil paint system using a painting knife [7] that is controlled using a haptic feedback device. Microsoft Surface [8] is based on the IR image capture of an IR illuminated diffuser screen underneath a transparent surface allowing natural finger-based interaction. Presence of real objects (e.g., brushes) can be detected, but only a blurred image of the brush is generated. Recently, Vandoren et al. presented a novel interactive canvas FluidPaint allowing the use of real wet brushes [9].

Animating. Regarding animation, only small initiatives have been taken so far. Van Laerhoven et al. introduce the concept of brushes and pigments enhanced with behavioural intelligence that allow the user to enrich and animate interactively created images by semiautonomously embedding procedural animation into them [10]. Some commercial digital art applications also claim to target at creating animations $([11,12,13])$, however, the animation part mainly is limited to the availability of a keyframe editor and the use of animated brushes to draw frames along a path. 


\begin{abstract}
Approach
In this section we give an overview of the main parts of our approach. We first describe the employed digital paint setup. Next, similar to the traditional process of previous section we will highlight both the painting and animation process.
\end{abstract}

\title{
Digital Paint Setup
}

For the simulation part, we make use of an interactive paint system that adopts physicallybased algorithms to simulate the complex interaction of a layered virtual canvas with different paint media, including watercolour, gouache and impasto, but also dry media like pencil or pastel [14]. This paint system encapsulates the drying process, paint diffusion as well as different pigment attributes (Figure 2(a)). Furthermore, it relies on Navier-Stokes for defining the velocities of the fluid body and on the specialised Kubelka-Munk colour mixing scheme for rendering paint layers. These features are vital elements to obtain the much sought after 'natural look' in digital artwork. Every step of the simulation makes full use of programmable graphics hardware to achieve interactive simulation rates.

Another important component of the system relates to its virtual brush design that relies on constrained energy optimisation and on an anisotropic friction model to deform the brush tuft and adequately translate the user's input (e.g., originating from a tablet interface) into realistic and predictable strokes. This design also allows for bidirectional pigment transfer between the canvas and the selected drawing tool. 
Regarding the interaction part, we chose to use a tangible interface to enable users to intuitively manipulate the drawing media (Figure 2(b)). The setup consists of an interactive multitouch surface based on the principle of frustrated total internal reflection (FTIR) [6]. The contact surface of each object on the paint table is tracked by a 0.3 megapixel video camera, equipped with an infrared band-pass filter, after which the footprint of the objects touching the surface is transferred to the paint simulation for further processing and rendering. In contrast to the original paint table, our setup is able to track multiple objects on the surface simultaneously. This way, artists can push pigment with multiple fingers at once, use special-purpose digital brushes with tufts, or even employ paper tissue.

A major advantage of this setup is that it provides coinciding action and perception spaces, resulting in a natural, reality-based methodology. In addition, it encourages multiple users to work collaboratively on the same artwork.

\section{Painting}

Starting from a blank canvas, the artist creates or alters an image by adding some kind of paint medium (e.g., gouache, impasto, watercolour, pastel) using fingers or several available tools (brushes, pencils, spatulas, sponges, fingers). A background image can be depicted as a reference and parts of the drawing canvas can interactively be masked to prevent paint from reaching or settling down on the masked parts. Drawings can also be separated into layers allowing for robust manipulations of the frame content such as depth ordering and hid- 
ing/revealing objects. Furthermore, users can interactively control aspects like drying time (including the possibility to keep paint permanent wet or pause the drying), undo mistakes, remove pigment and water as desired and have the ability to save, load or reuse intermediate results.

Watercolour images made with the system contain the typical effects that can be recognised in images produced with real thin paint, like the dark-edge effect, watercolour glazing, wet-on-wet painting and the use of different pigment types.

\section{Animating}

While animating, each subsequent frame in the animation is created by pushing the drawing medium of the artwork in the current frame towards its desired position for the next frame. During interaction, movement on the multitouch surface is converted to a vector field based on the current velocity of the brush/finger (see Figure 3(a)), which is then applied to the paint (both pigment and water) by means of an advection step $-(\vec{v} \cdot \nabla) p$, where $\vec{v}$ denotes the 2D velocity vector field and $p$ the amount of paint at a certain position on the canvas. As in our paint system mass is contained in the grid cells of the digital canvas, the advection scheme boils down to individual cells exchanging content with their neighbours (Figure 3(b)) [14]. For each cell, we measure the volume of paint that is exchanged with all neighbouring cells as follows.

When calculating the amount of mass that flows from the centre cell to its right neigh- 
bour with velocities $\vec{v}_{\text {center }}$ and $\vec{v}_{\text {right }}$ respectively, as shown in Figure 3(b) (left), any point along this border has velocity:

$$
\vec{v}_{\text {average }}=\frac{\vec{v}_{\text {center }}+\vec{v}_{\text {right }}}{2}
$$

Within a given time step $\Delta t$, a distance $\Delta x=\left(\vec{v}_{\text {average }}\right)_{x} \Delta t$ is travelled. In case we are calculating the movement of water, the area covered by the volume of displaced water is given by the coloured area in Figure 3(b) (left), and equals $\Delta x$ (cellheight). The total volume of displaced water then is $\Delta V=\Delta x$ (cellheight) $w_{\mathrm{i}, \mathrm{j}}$ (Figure 3(b) (right)). The change in water quantity is given by the following equation:

$$
\Delta w=\frac{\Delta V}{(\text { cellwidth } \times \text { cellheight })}
$$

This procedure is repeated to calculate the exchanged water quantities with the three remaining neighbours. We add up the results and divide it by four, because each neighbour contributes exactly one quarter to the total flux. The same approach can be followed to work out the movement of pigment.

Figure 4 depicts some stills of a user pushing paint (in this case impasto) with two fingers on an interactive multitouch surface.

In order to assure more control over the way the artist is obliged to make things move, he/she can rely on digital animation tools to make manipulative operations more predictable 
including a keyframe editor, onion skinning and a flipbook tool (Figure 5). The onion skinning tool aids the artist to achieve good frame-to-frame coherence, resulting in smoother frame transitions by showing trace layers of previously painted frames whereas the digital flipbook tool provides immediate feedback on the status of the animation being created.

\section{Results}

Figure 7 depicts some snapshots of a paint-on-glass animation of a bird created with impasto paint illustrating the stretch and smear effects. Even for the start frame, the artist chose to gradually refine a basic image (painted using a brush) using his fingers only (see Figure 6). The entire animation consists of 31 frames of which each is a modified version of its predecessor by only pushing paint from frame to frame.

Discussion. The target audience of our setup is skilled artists as the paint-on-glass technique itself requires some experience in the field of painting. The platform itself is targeted at both traditional and digital artists as the interface is similar to the traditional environment.

Therefore traditional as well as digital painters were involved in an informal user test evaluating the setup as well as creating the examples. Only basic instructions were given (i.e. explaining the user interface). After that, the test participants were allowed to work on their subjects of choice without any training.

All participants worked between two and three hours to complete their animations (43 
frames on average) which is significantly faster than the traditional way of working (i.e. around 20 frames a day, on average). Although the test session was rather short we can say that all participants were very positive. They found the user interface familiar and praised the real-life behaviour of manipulating the paint as well as interacting with the setup, resulting in a natural and reality-based methodology.

\section{Conclusions}

In this paper we dealt with making animated films by pushing around wet media directly under the camera, known as paint-on-glass animation. Without the ability to rehearse and refine the animation as opposed to classic $2 \mathrm{D}$ animation, this particular form of animation is a painstaking process for which animators need to persevere regardless incorporating any errors or unwanted smudging into the sequence.

Our approach tackles the issues inherent to paint-on-glass animation by offering the animator an interactive paint system that adopts physically-based algorithms to simulate the complex interaction of a virtual canvas with different paint media, and a set of digital tools that do enable him/her to achieve the fine quality of a finished painting for each frame while preserving all artisan control. Regarding the interaction part, a tangible interface was chosen to enable users to intuitively manipulate the drawing media using their own hands. 
Future Work. The emphasis in this paper is on paint-on-glass animation. However, the same approach could be applied to any "under-the-camera-technique" such as animating using fine sand, sugar or coffee grind. For the simulation part this requires a bidirectional transfer of particles; particles need to be deposited or scraped off again. Regarding the interaction process we have to consider how to sprinkle dry media.

Furthermore, we strongly believe in investigating new interaction techniques which are not present at all in traditional animation such as using simple hand gestures and finger taps to easily navigate between frames or assets. Inspired by ShapeTouch [15] another option is to exploit the shape of hands to work on the interactive surface just like when dealing with real physical objects (e.g., pinning, peeling or flicking).

\section{Acknowledgements}

We gratefully express our gratitude to the European Fund for Regional Development and the Flemish Government, and to Xemi Morales and Karel Robert for their artistic contribution.

\section{References}

[1] Aleksandr Petrov. World Wide Web, http://en.wikipedia.org/wiki/Aleksandr_Petrov_

[2] Suguru Saito and Masayuki Nakajima. 3D physics-based brush model for painting. In Proceedings of SIGGRAPH Conference abstracts and applications, page 226. ACM, 
1999.

[3] W. V. Baxter, V. Scheib, M. C. Lin, and D. Manocha. DAB: Interactive haptic painting with 3D virtual brushes. In Proceedings of SIGGRAPH, pages 461-468. ACM, 2001.

[4] Nelson Chu and Chiew-Lan Tai. Real-time painting with an expressive virtual Chinese brush. IEEE Computer Graphics and Applications, 24(5):76-85, 2004.

[5] Tom Van Laerhoven and Frank Van Reeth. Brush up your painting skills: Realistic brush design for interactive painting applications. The Visual Computer. Computer Graphics International (CGI2007), 23:763-771, September 2007.

[6] Peter Vandoren, Tom Van Laerhoven, Luc Claesen, Johannes Taelman, Chris Raymaekers, and Frank Van Reeth. IntuPaint: Bridging the gap between physical and digital painting. In Proceedings of TABLETOP 2008, IEEE International Workshop on Horizontal Interactive Human Computer System, pages 71-78, October 2008.

[7] N. Okaichi, H. Johan, T. Imagire, and T. Nishita. A virtual painting knife. The Visual Computer. Computer Graphics International (CGI2008), pages 753-763, 2008.

[8] Microsoft. Microsoft surface. World Wide Web, http://www.microsoft.com/surface/.

[9] Peter Vandoren, Luc Claesen, Tom Van Laerhoven, Johannes Taelman, Chris Raymaekers, Eddy Flerackers, and Frank Van Reeth. FluidPaint: an interactive digital 
painting system using real wet brushes. In Proceedings of ITS 2009, ACM ACM International Conference on Interactive Tabletops and Surfaces, pages 53-56, November 2009.

[10] Tom Van Laerhoven, Fabian Di Fiore, and Frank Van Reeth. Hand-painted animation with intelligent brushes. Computer Animation and Virtual Worlds. Computer Animation and Social Agents (CASA2008), pages 365-374, 2008.

[11] Corel Painter X. World Wide Web, http://www. corel.com/.

[12] Project Dogwaffle. World Wide Web, http: / / www . thebest 3d. com/dogwaffle/.

[13] Adobe PhotoShop. World Wide Web, http: //www . photoshop.com/.

[14] Tom Van Laerhoven and Frank Van Reeth. Real-time simulation of watery paint. In Journal of Computer Animation and Virtual Worlds (Special Issue CASA 2005), volume 16:3-4, pages 429-439. J. Wiley \& Sons, Ltd., October 2005.

[15] Xiang Cao, Andrew Wilson, Ravin Balakrishnan, Ken Hinckley, and Scott Hudson. ShapeTouch: Leveraging contact shape on interactive surfaces. In Proceedings of TABLETOP 2008, IEEE International Workshop on Horizontal Interactive Human Computer System, pages 139-146, October 2008. 


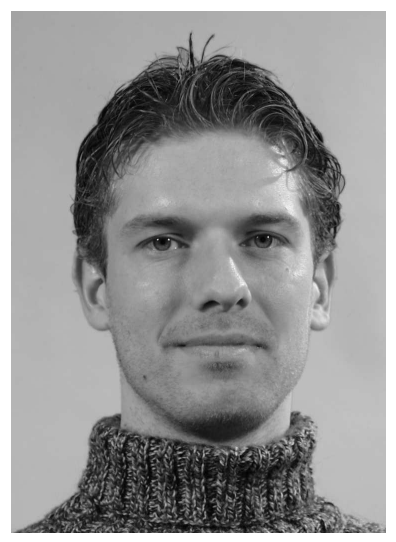

dr. Tom Van Laerhoven is a senior researcher in computer science at Hasselt University (UHasselt) in Diepenbeek, Belgium. He obtained a MS in computer science in 2000 at UHasselt. In 2006, he finished his $\mathrm{PhD}$ entitled "An Extensible Simulation Framework Supporting Physically-based Interactive Painting" at the Expertise Centre for Digital Media (EDM), a research institute of Hasselt University. His research activities are concerned with computer animation, physically-based modelling and animation, non-photorealistic rendering and parallel and distributed algorithms.

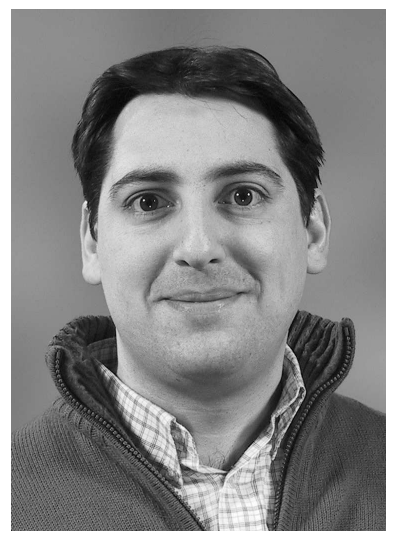

Prof. dr. Fabian Di Fiore is an associate professor in computer science at Hasselt University (UHasselt) in Belgium. He obtained a MS in computer science in 1997 at the University of Leuven. In 2004, he finished his $\mathrm{PhD}$ entitled "A 2.5D Modelling and Animation Framework Supporting Computer Assisted Traditional Animation" at the Expertise Centre for Digital Media (EDM), a research institute of Hasselt University. His research activities are concerned with computer animation, nonphotorealistic rendering and animation, and highly stylised drawn animation. 


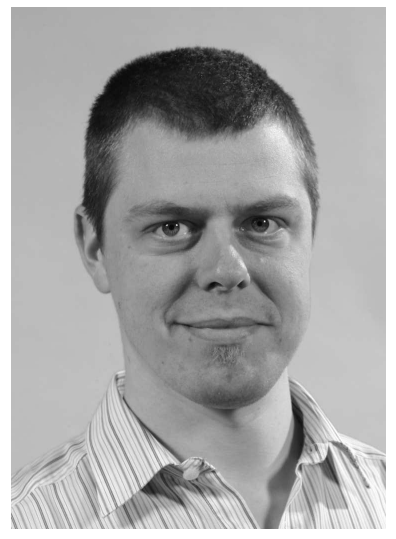

dr. William Van Haevre is a senior researcher in computer science at Hasselt University (UHasselt) in Diepenbeek, Belgium. He obtained a MS in computer science in 2000 at UHasselt. In 2007, he finished his $\mathrm{PhD}$ entitled "Realism in Environment Sensitive Plant Models and their Animation" at the Expertise Centre for Digital Media (EDM), a research institute of Hasselt University. His research interests include computer graphics and animation, physically-based modelling and animation of natural phenomena, video-based animation of plants and simulating highly stylised drawing techniques.

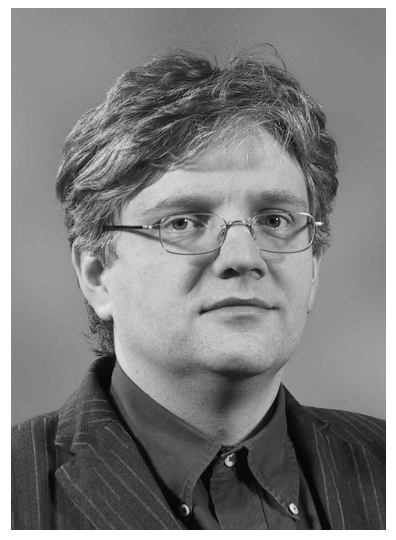

Prof. dr. Frank Van Reeth is a full-time professor of Computer Science at Hasselt University (UHasselt) in Belgium and Deputy Managing Director of the Expertise centre for Digital Media (EDM), a research institute of Hasselt University. He is co-founder of seven spin-off companies and a member of the board of ANDROME NV. His research interests include computer graphics, computer animation, virtual environments, multimedia technology and telematics. He was project leader of several industrial contract research projects, technical representative as well as project manager in various EC research projects, and involved in several IBBT projects. He acted as evaluator of national and European R\&D proposals involving the above research domains. He is a member of Eurographics, ACM, VRS, IEEE and CGS. 
1

2

3

4

5

6

7

8

9

10

11

12

13

14

15

16

17

18

19

20

21

22

23

24

25

26

27

28

29

30

31

32

33

34

35

36

37

38

39

40

41

42

43

44

45

46

47

48

49

50

51

52

53

54

55

56

57

58

59

60

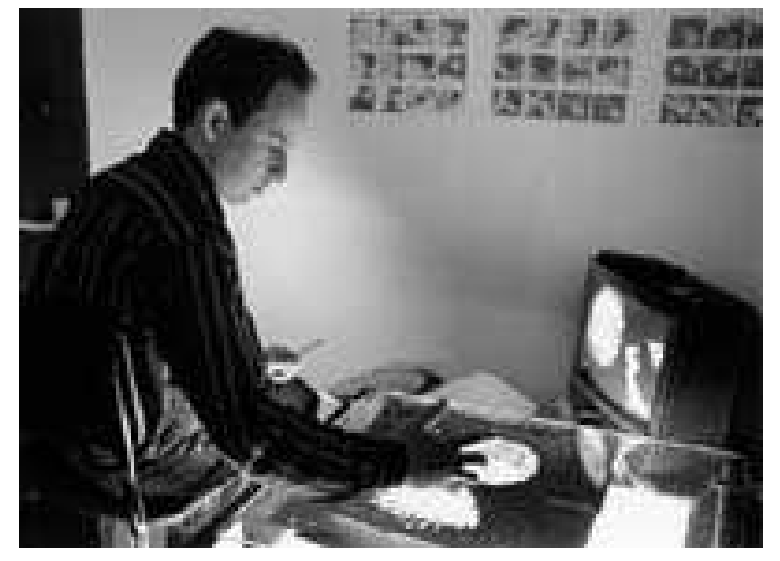

Figure 1: An artist at work using an "under-the-camera-technique" with a combination of sand and ink (c) Gerald Conn. 


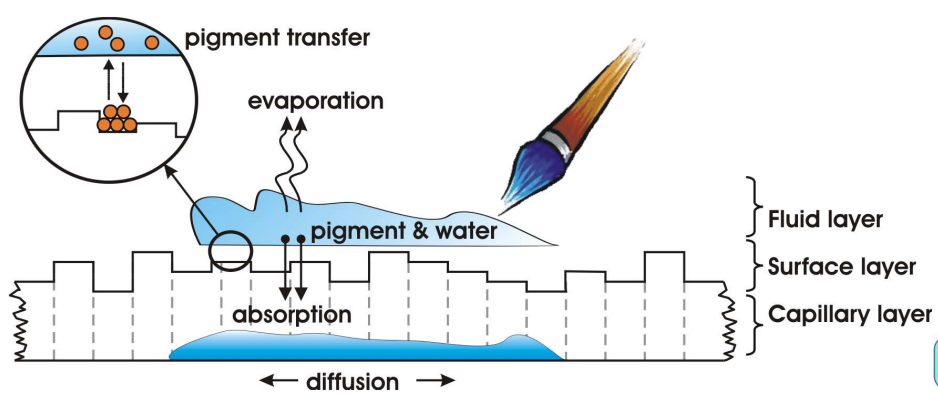

(a)

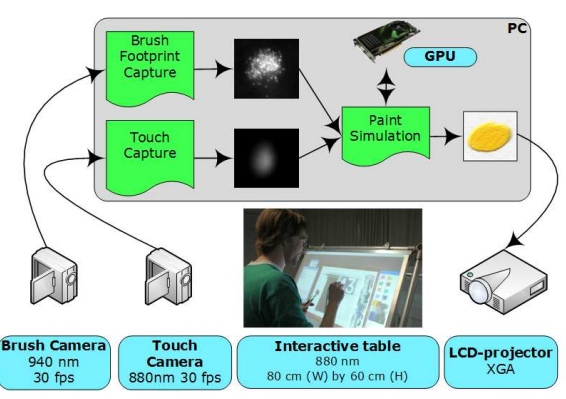

(b)

Figure 2: a) Physically-based paint simulation. b) The setup includes an interactive surface on which the user can manipulate paint with multiple fingers at once, or using specialpurpose tools. 


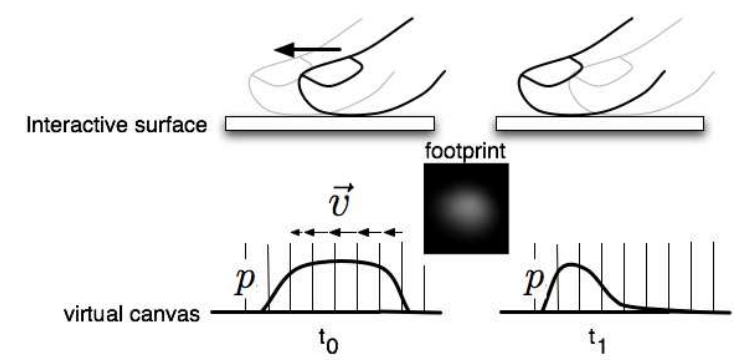

(a)
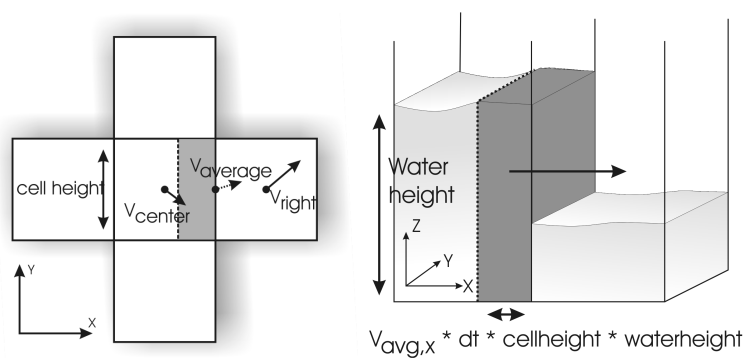

(b)

Figure 3: a) Pushing paint results in a velocity field that locally manipulates the exchange of pigment quantities. b) Moving water to a right neighbouring cell. The dark areas represent the volume of displaced water. 

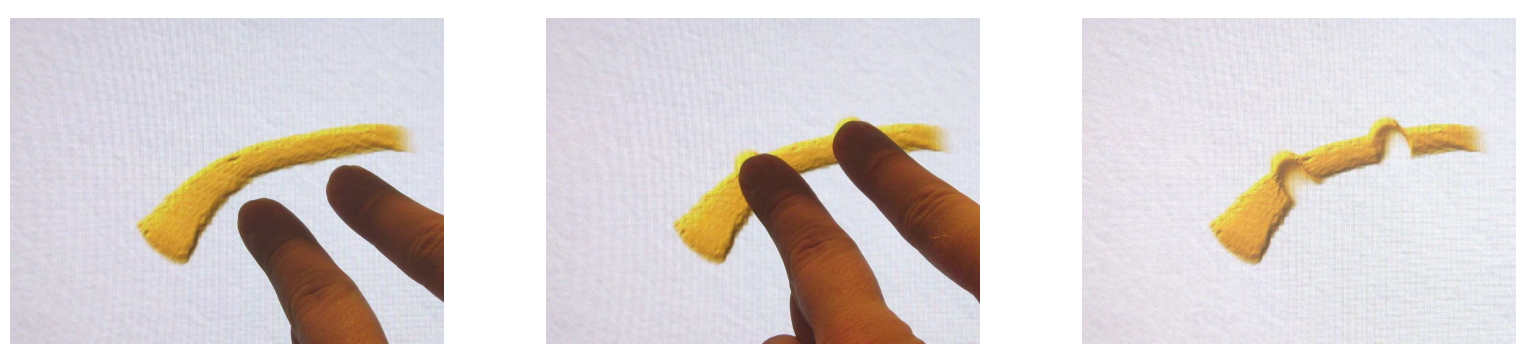

Figure 4: Some stills depicting a user pushing paint (impasto) with two fingers on an interactive multitouch surface. 

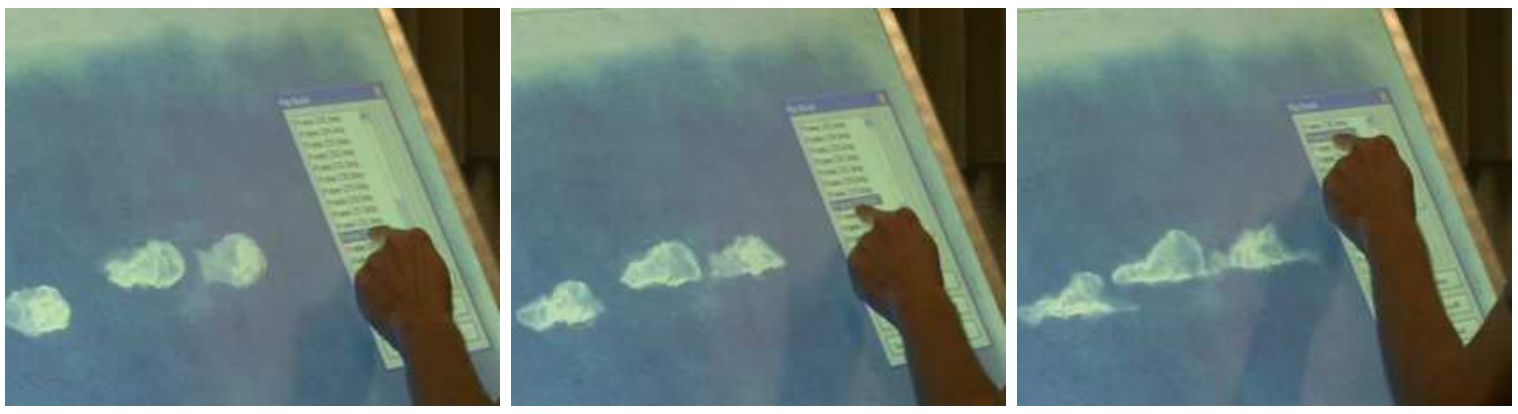

Figure 5: When drawing a new frame the animator can check temporal coherence by flipping through all previous frames. 

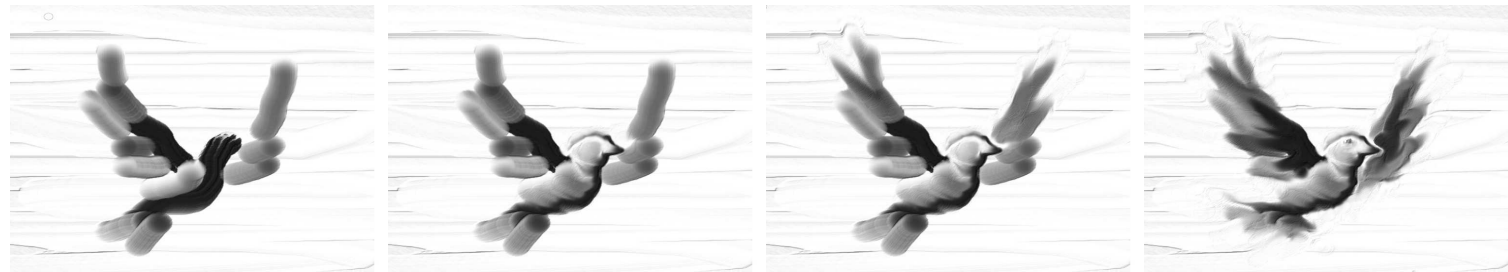

Figure 6: The making of an entire new frame (last image) from scratch by gradually refining a basic image (first image) using fingers only. 


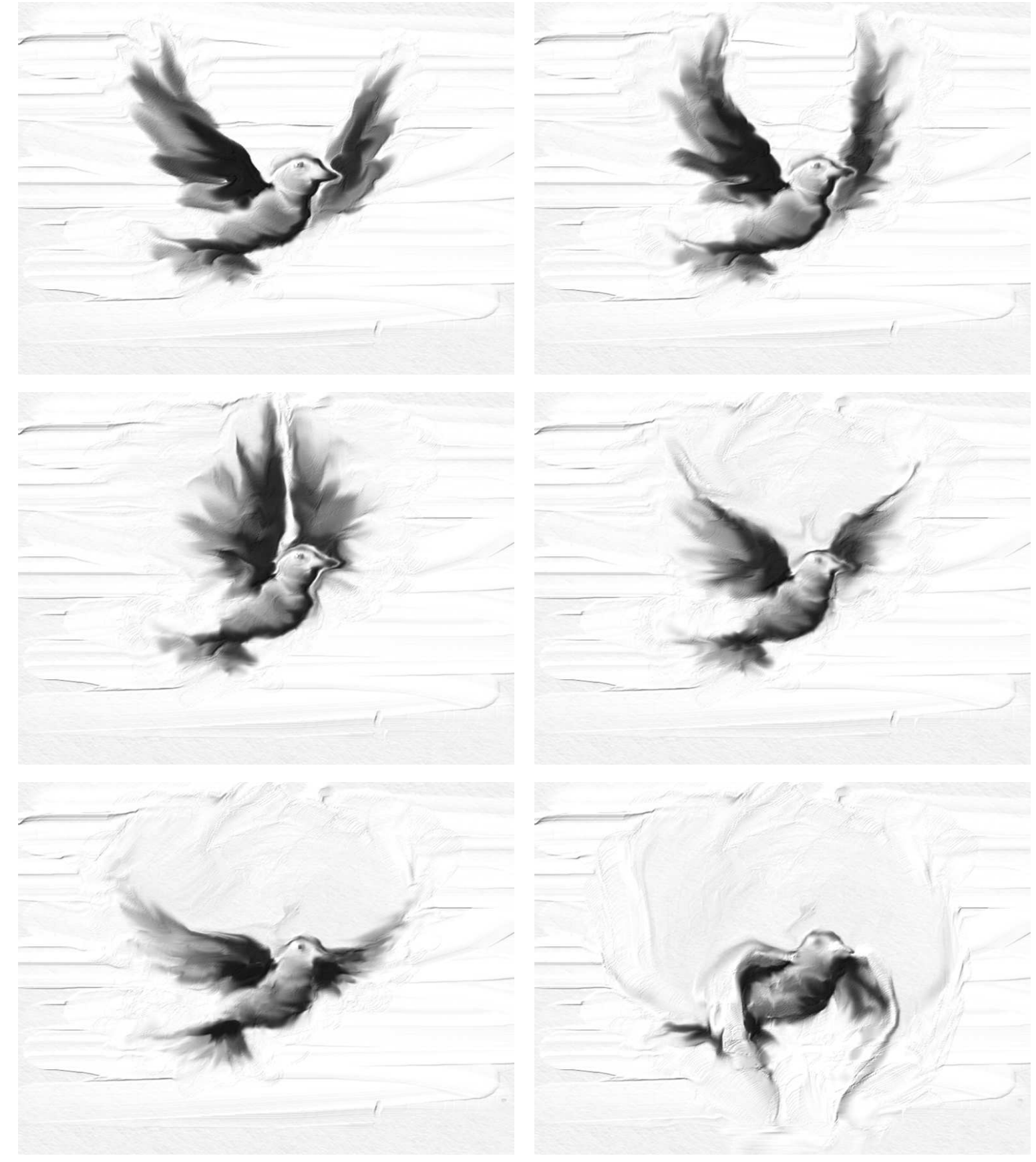

Figure 7: Some snapshots of a paint-on-glass animation of a bird created with impasto paint. Each image is a modified version of its predecessor by only pushing paint from frame to frame. 